\title{
Implementation of Bessel's method for solar eclipses prediction in the WRF-ARW model
}

\author{
Alex Montornès ${ }^{1}$, Bernat Codina ${ }^{1}$, John W. Zack ${ }^{2}$, and Yolanda Sola ${ }^{1}$ \\ ${ }^{1}$ Department of Astronomy and Meteorology, University of Barcelona, Barcelona, Spain \\ ${ }^{2}$ MESO Inc., Troy, USA \\ Correspondence to: Alex Montornès (amontornes@am.ub.es)
}

Received: 3 October 2015 - Published in Atmos. Chem. Phys. Discuss.: 18 January 2016

Revised: 17 April 2016 - Accepted: 23 April 2016 - Published: 17 May 2016

\begin{abstract}
Solar eclipses are predictable astronomical events that abruptly reduce the incoming solar radiation into the Earth's atmosphere, which frequently results in nonnegligible changes in meteorological fields. The meteorological impacts of these events have been analyzed in many studies since the late 1960s. The recent growth in the solar energy industry has greatly increased the interest in providing more detail in the modeling of solar radiation variations in numerical weather prediction (NWP) models for the use in solar resource assessment and forecasting applications. The significant impact of the recent partial and total solar eclipses that occurred in the USA (23 October 2014) and Europe (20 March 2015) on solar power generation have provided additional motivation and interest for including these astronomical events in the current solar parameterizations.

Although some studies added solar eclipse episodes within NWP codes in the 1990s and 2000s, they used eclipse parameterizations designed for a particular case study. In contrast to these earlier implementations, this paper documents a new package for the Weather Research and ForecastingAdvanced Research WRF (WRF-ARW) model that can simulate any partial, total or hybrid solar eclipse for the period 1950 to 2050 and is also extensible to a longer period. The algorithm analytically computes the trajectory of the Moon's shadow and the degree of obscuration of the solar disk at each grid point of the domain based on Bessel's method and the Five Millennium Catalog of Solar Eclipses provided by NASA, with a negligible computational time. Then, the incoming radiation is modified accordingly at each grid point of the domain.

This contribution is divided in three parts. First, the implementation of Bessel's method is validated for solar eclipses
\end{abstract}

in the period 1950-2050, by comparing the shadow trajectory with values provided by NASA. Latitude and longitude are determined with a bias lower than $5 \times 10^{-3}$ degrees (i.e., $\sim 550 \mathrm{~m}$ at the Equator) and are slightly overestimated and underestimated, respectively. The second part includes a validation of the simulated global horizontal irradiance (GHI) for four total solar eclipses with measurements from the Baseline Surface Radiation Network (BSRN). The results show an improvement in mean absolute error (MAE) from 77 to $90 \%$ under cloudless skies. Lower agreement between modeled and measured GHI is observed under cloudy conditions because the effect of clouds is not included in the simulations for a better analysis of the eclipse outcomes. Finally, an introductory discussion of eclipse-induced perturbations in the surface meteorological fields (e.g., temperature, wind speed) is provided by comparing the WRF-eclipse outcomes with control simulations.

\section{Introduction}

Solar eclipses are predictable astronomical events that momentarily reduce the incoming radiation to the Earth's atmosphere, inducing a significant change in the meteorological fields. The impact of the shadow of the Moon on the Earth's atmosphere has awakened the interest of many scientists since the second part of the 20th century. Solar eclipse episodes are excellent experiments for analyzing the response of the atmosphere (e.g., surface and planetary boundary layer, PBL) and for testing the response of the physical schemes in numerical weather prediction (NWP) models. During a solar eclipse, the region under the shadow experi- 
ences a similar physical process to that which occurs at sunrise and sunset but abruptly and on a shorter timescale (Anderson, 1999).

The first modern studies related to the relationship between the atmosphere and the solar eclipses appeared in the late 1960s mainly focused on the ozone variations and their impact on the stratosphere and mesosphere (Bojkov, 1968; Randhawa, 1968; Ballard et al., 1969).

At the beginning of the 1970s, Chimonas and Hines (1970, 1971) suggested that the cooling produced by the lunar shadow, crossing the atmosphere of the Earth at supersonic speeds, should produce gravity waves in the upper layers, measurable as surface pressure fluctuations. Based on this discussion, many studies were published that tried to detect these waves such as Davis and Da Rosa (1970), Anderson et al. (1972) and Chimonas (1973) or later publications such as Fritts and Luo (1993), Altadill et al. (2001) and Zerefos et al. (2007).

Although several early studies (e.g., Stewart and Rouse, 1974; Antonia et al., 1979) examined the impact of solar eclipses on surface processes, it was not until the late 1990s and early 2000s that the focus shifted to the variations in temperature, humidity, wind speed, turbulence and atmospheric chemistry. Fernández et al. (1993a, b) analyzed the variations produced by the total eclipse of 11 July 1991 on different meteorological fields using a set of surface stations and radio soundings for different sites in Costa Rica. The first study concerned the impact on the global horizontal irradiance (GHI) measurements, while the second one analyzed the effect on temperature, humidity and wind speed on the ground and in the free atmosphere. Fernández et al. (1993b) observed negative temperature deviations ranging from 2 to $5{ }^{\circ} \mathrm{C}$ reaching the minimum value between 10 and $30 \mathrm{~min}$ after the maximum obscuration of the solar disk. Surface wind speed experienced a noteworthy reduction some minutes after reaching the lowest temperature in those sites not dominated by the large-scale patterns. In the free atmosphere, the highest temperature and wind speed variations were observed at $\sim 13 \mathrm{~km}$ (i.e., $175 \mathrm{hPa}$ ), with thermal differences from -2 to $-6^{\circ} \mathrm{C}$ and a high deviation in wind direction compared with soundings on similar days. Fernández et al. (1996), Segal et al. (1996) and Anderson (1999), among others, reported similar results, focusing on surface temperature. Meanwhile, Eaton et al. (1997) examined the effects of a solar eclipse on the PBL using the episode of 10 May 1994 for the study. The analysis showed a clear impact on the heat exchange (sensible and latent), a reduction in the turbulence processes and a significant negative deviation in the refractive index structure parameter. Moreover, using a frequency-modulated, continuous-wave (FM-CW) radar operating at $2.9 \mathrm{GHz}$, Eaton et al. (1997) reported the development of Kelvin-Helmholtz waves during the eclipse.

The most meteorologically analyzed eclipse event is the total solar eclipse that occurred over Europe on $11 \mathrm{Au}-$ gust 1999. The expanded use of mesoscale NWP models dur- ing the late 1990s along with the dense network of weather stations across Europe facilitated a large number of publications on this event. In general, these studies focused on the impact on near-surface variables. For example, Hanna (2000) analyzed the measured variation at stations across the United Kingdom, while Aplin and Harrison (2003) provided a broader-scale analysis of the variations across the continent. Other relevant studies in other areas of interest were performed by Abram et al. (2000), Zerefos et al. (2001) and Anfossi et al. (2004), among others. Abram et al. (2000) measured the effect of the solar reduction on the tropospheric chemistry, particularly on the hydroxyl radical and ozone in England. Zerefos et al. (2001) examined the induced thermal fluctuations in the ozone layer, ionosphere and troposphere at stations in the Balkans, observing the existence of dominant oscillations in the parameters related to the ionosphere and the ozone layer. Anfossi et al. (2004) used a three-axis propeller anemometer (Gill-type) and a fast-response temperature sensor in a mast located in France for measuring the turbulence variation during the eclipse. In that study, they documented a rapid turbulent kinetic energy decay in time.

More recently, other studies such as Founda et al. (2007) and Gerasopoulos et al. (2008) have been focused on the total solar eclipse which occurred on 29 March 2006 over eastern Europe and particularly in Greece. Subrahamanyam et al. (2011) analyzed the behavior of the atmospheric surface layer, comparing the eclipse observations for the event on 15 January 2010, with similar measurements recorded on non-eclipse cloudless days in India used as a baseline.

Given the low frequency of solar eclipses in regions with meteorological stations, atmospheric models are suitable tools for analyzing the response of the atmosphere during a solar eclipse episode. The first studies with models appeared during the 1990s. Segal et al. (1996) used a boundary layer version of the model described in Arritt (1989) in order to evaluate the spatial and temporal effects on shelter temperature using the total solar eclipse of 10 May 1994. The general features of the eclipse were quantified using data from the ephemerides and then refined by direct computation of the Sun and Moon geometry based on standard methods of celestial mechanics with a suitable accuracy. Gross and Hense (1999) presented an NWP model study using the Deutschland-Modell (DM) from the German Weather Service (DWD) for analyzing the meteorological effects of the 11 August 1999 eclipse. In this case, the episode was parameterized in terms of the shadow's trajectory and approximating the reduction in the solar insolation. The solar constant $S_{0}$ was modified as $0.01 S_{0}$ in the center and assuming a linear increase in the north-south direction. This parameterization produced a sufficient accuracy for the purposes of the experiment with $5 \mathrm{~min}$ in time and $10 \%$ in the amplitude of the eclipse. Vogel et al. (2001) used the Karlsruhe Atmospheric Mesoscale Model (KAMM; Adrian and Fiedler, 1991) for studying the perturbation in temperature and wind driven by the eclipse of 11 August 1999 in southern Ger- 
many. In this case, the solar constant was modified using a mathematical expression referred as "obscuration function" derived from geometric relationships in terms of the solar, lunar and observer positions. Zanis et al. (2001) used a simple photochemical box model for investigating the response of the tropospheric ozone variations during a photolytical perturbation as in the case of that European eclipse. Szałowski (2002) built a basic model of local soil and air temperature changes in Poland for the same episode. The solar obscuration was evaluated following geometric relationships as a function of the topocentric coordinates of the centers of the solar and lunar disks in the equinoctial system and the angular radii of both celestial bodies. Eckermann et al. (2007) investigated the atmospheric response to the total solar eclipse of 4 December 2002 with the high-altitude global NWP model (Navy's Operational Global Atmospheric Predictions System - Advanced-Level Physics, High-Altitude, NOGAPS-ALPHA). In this experiment, the obscuration of the solar disk was evaluated assuming a linear variation from the center of the eclipse to the penumbra region. Related to the Weather Research and Forecasting-Advanced Research WRF (WRF-ARW) model, Founda et al. (2007) parameterized the eclipse of 29 March 2006 assuming a variation in the solar constant proportional to the distance from the shadow axis, considered as a point moving on Earth with a specific velocity. More recently, Wu et al. (2011) used the WRFARW coupled with the WRF-CHEM module for analyzing the sensitivity of the tropospheric ozone and other chemical species as well as the effects on meteorological variables to the limb darkening effect as well as the effects on meteorological and other chemical species during the eclipse of 22 July 2009 over China. The solar eclipse effect was added, modifying the solar radiation and photolysis rates using a scaling factor as a function of the latitude, longitude, time and wavelength. The degree of obscuration was evaluated as proportional to the distance to the center of the total eclipse track provided by NASA.

The recent growth in the solar energy industry has greatly increased the interest in including more detail in the modeling of solar irradiance variations in NWP models for the use in solar resource assessment and forecasting applications. Regarding the resource forecasting, solar eclipses are episodes that increase errors significantly because the operational shortwave schemes implemented within NWP models neglect these astronomical events. We propose a general approach for modeling the eclipse effects within the WRFARW model based on Bessel's method (e.g., Chauvenet, 1871) and the Five Millennium Catalog of Solar Eclipses provided by NASA (Espenak and Meeus, 2008). The method is widely used in many astronomical applications related to occultations and eclipses as a particular case. This approach replaces the highly complex equations describing the orbital motions of the Sun, Moon and Earth with a simpler equation set expressed in terms of the location on the Earth's surface and the position and motion of the Moon's penumbral and umbral shadows with respect to the center of the Earth. The use of this simpler description does not lose accuracy and it becomes independent of the observer coordinates. These variables are used to evaluate the eclipse conditions at each grid point represented by the degree of obscuration and modifying the incoming radiation accordingly.

The study has two major components. In the first part, the algorithm implemented to model solar eclipses in the WRFARW is described in Sect. 2 and the results of a validation of the solar eclipse trajectories computed by the algorithm with respect to published NASA values are presented in Sect. 3 . The second component of the paper presents results from tests of the new WRF-ARW algorithm and code in simulations of four eclipse cases. The validation of the simulated global horizontal irradiance with data from the Baseline Surface Radiation Network (BSRN; Ohmura et al., 1998) is provided in Sect. 4 and the simulated eclipse-induced temperature as well as wind speed and direction perturbations are described in Sect. 5.

\section{Implementation in the WRF-ARW model}

The conceptual idea for including the solar eclipses within the WRF-ARW model is similar to the previous attempts performed by Gross and Hense (1999) or Founda et al. (2007), among others, as we have explained in the "Introduction". The solar eclipse occurs when the disk of the Sun is hidden partially or totally by the Moon. Consequently, this process produces a reduction in the incoming radiation at the top of the atmosphere (TOA), $S_{\text {in }}$.

The magnitude is computed in terms of the solar constant, $S_{0}$, and the cosine of the solar zenith angle, $\mu_{0}$, as

$S_{\text {in }}=S_{0} \mu_{0}$.

In the default version of the WRF-ARW model, $S_{0}$ is evaluated at the module named "radiation_driver" at each radiative call and shared with all shortwave parameterization routines as an input variable. Given one day of the year, this variable is assumed as a constant at all grid points of the domain (i.e., scalar magnitude). This number is determined using a baseline solar constant of $1370 \mathrm{~W} \mathrm{~m}^{-2}$ modulated by an eccentricity factor, determined following the methodology of Paltridge and Platt (1976) as a function of the day of the year. On the other hand, $\mu_{0}$ is calculated using spherical astronomy equations in terms of the date, time and the geographic coordinates of the current grid point. Therefore, Eq. (1) has a dependence on grid point $i, j$ and on time $t$ as

$S_{\mathrm{in}, t i j}=S_{0, t} \mu_{0, t i j}$.

As discussed in previous publications such as Founda et al. (2007), the key point in modeling the impact of solar eclipses in the WRF-ARW radiation physics is the modification of 
the incoming radiation by a spatially dependent (i.e., a 2-D array) degree of obscuration $D$. This variable takes into account the part of the solar disk that is hidden by the Moon with a geographical and temporal dependence due to the observer perspective (i.e., position within the model domain) and the solar and lunar motions with respect to the Earth. Thus, given one time $t$, we can rewrite Eq. (2) as

$S_{\mathrm{in}, t i j}=S_{0, t} \mu_{0, t i j}\left(1-D_{t i j}\right)$.

When the grid point is not under eclipse conditions, $D_{t i j}=$ 0 and Eq. (3) becomes Eq. (2). By contrast, when a grid point is under the totality, $D_{t i j}=1$ and $S_{\mathrm{in}, t i j}$ becomes zero.

The eclipse trajectory is determined using Bessel's method broadly explained in several manuals, such as Chauvenet (1871), and briefly presented in Appendix A for readers with some astronomical background. Although this approach dates from the 19th century, it is still used by many institutions, such as NASA.

Bessel's method is a general approach used to predict the place and time for observing all celestial phenomena as occultations and eclipses. In the case of the solar eclipses, this approach projects the Sun and Moon orbit trajectories onto a plane passing through the Earth's center and perpendicular to the axis of the Moon's shadow; this plane is defined as the "fundamental plane". On it, a Cartesian coordinate system in $\mathbb{R}^{3}$ is used, with the $X$ and $Y$ axes constructed on the fundamental plane and with the origin at the Earth's center. By construction the $Z$ axis is normal to the fundamental plane and parallel to the axis of the shadow. This new reference system is useful because we can define a set of variables that are only relative to the fundamental plane and invariant to the observer. These magnitudes are denoted as "Besselian elements" and they are detailed in Appendix A. As the Besselian elements only depend on the fundamental plane and the astronomical ephemeride or almanac, they can be evaluated before an eclipse without considering the point of view of the observer.

There are several catalogs for eclipses and occultations providing the Besselian elements based on the astronomical ephemerides. For the particular case of solar eclipses, NASA provides two catalogs: the Five Millennium Catalog of Solar Eclipses (Espenak and Meeus, 2008) that contains all partial, annular, total and hybrid eclipses from 2000 BCE to 3000 CE and the Ten Millennium Catalog of Long Solar Eclipses (Espenak and Meeus, 2009) with a period from 4000 BCE to $6000 \mathrm{CE}$.

We store these Besselian elements in a WRF file named "eclipse_besselian_elements.dat" that must be present in the running folder for the model. This file contains a database of all partial, annular, hybrid and total eclipses from 1950 to 2050 (both included) based on the Five Millennium Catalog of Solar Eclipses (Espenak and Meeus, 2008).

Following the set of equations described in Appendix A, the degree of obscuration is evaluated for each grid point at each radiation call. Then, the incoming radiation is modified following Eq. (3) before calling the configured solar parameterization.

\section{Algorithm validation}

In order to evaluate the degree of accuracy and reliability in the eclipse computation, the proposed algorithm has been validated with respect to NASA's values (Espenak and Meeus, 2008). As the lunar shadow has a circular shape (Eq. A17) in which each Earth point is separated by a distance $\Delta$ from the center, the evaluation of the shadow's axis is enough for determining the degree of accuracy of the new algorithm.

The validation includes all total, annular and hybrid episodes for the period between 1950 and 2050. Partial eclipses cannot be validated because the trajectory is not well-defined on the Earth's surface (Appendix A). Moreover, there are some particular cases near the poles in which the axis of the shadow does not cross the Earth's surface and hence they are mathematically undefined. The following instances of such cases are not included in the validation: 30 April 1957 (annular, Northern Hemisphere), 23 October 1957 (total, Southern Hemisphere), 2 November 1967 (total, Southern Hemisphere), 29 April 2014 (annular, Southern Hemisphere), 9 April 2043 (total, Northern Hemisphere) and 3 October 2043 (annular, Southern Hemisphere).

The results show a bias less than $\pm 5 \times 10^{-3}$ degrees for latitude and longitude, and in many cases even lower than $\pm 1 \times 10^{-4}$ degrees (Fig. 1), with the errors in longitude being significantly higher than in latitude. In general, latitude shows positive biases, while longitude tends to be underestimated in other words, the modeled eclipse experiences a small temporal delay with respect to the NASA values. There are no relevant differences between eclipse types.

These differences are most likely associated with the variations in the accuracy of physical constants and the precision of the calculations due to compiler and coding language differences. On the one hand, the results by using double precision instead of single precision show a near-zero bias in latitude, while in longitude the bias is reduced by a factor of 5 (i.e., $110 \mathrm{~m}$ as a maximum). For economy these results are not included in the present study. Furthermore, there are two constants that may disturb the result: (i) the Earth's eccentricity and (ii) the correcting factor for the true longitude considering the nonuniform rotation of the Earth (Eq. A35).

The degree of accuracy shown in Fig. 1 (i.e., bias less than $\pm 550 \mathrm{~m}$ in the Equator and decreasing with latitude) is enough for most of the mesoscale applications. Moreover, the potential improvement added by features described in the previous paragraph does not justify the increase in memory resources. 


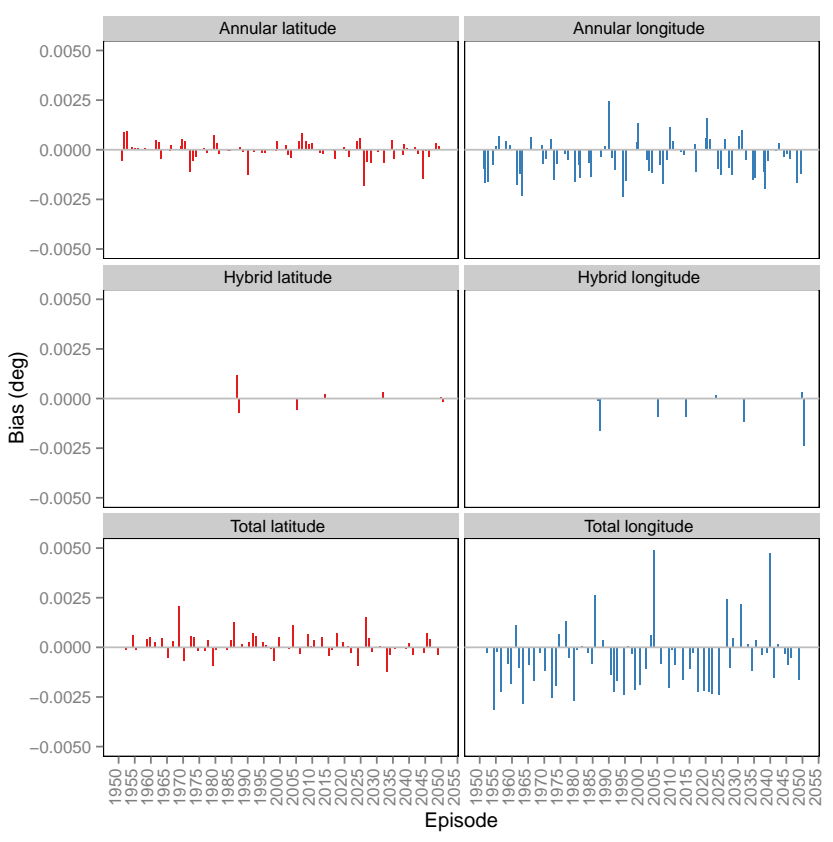

Figure 1. Bias in the eclipse's track computed by WRF-ARW in comparison to the NASA values.

\section{Case studies}

The proposed implementation within the WRF-ARW model is tested in four real simulations with two goals: (i) to evaluate the degree of improvement in the GHI outcomes required for solar energy industry applications and (ii) to observe the degree of realism of the WRF-ARW model response necessary for future scientific research in the line of previous works such as Founda et al. (2007).

As real measurements, we use data from the BSRN network (Ohmura et al., 1998) because this data set provides radiation, surface and upper-air measurements for 58 stations around the world in many climate zones and, in some cases, covering periods longer than 20 years. Moreover, the solar radiation measurements are provided with a high time resolution (1 to $3 \mathrm{~min}$ ), which is convenient for evaluating GHI performance.

The case studies are chosen in terms of the spatial and temporal coverage of the BSRN stations and the data availability. After a previous analysis of the data sets, four total-eclipse episodes are presented: 3 November 1994 (South America), 11 August 1999 (Europe), 29 March 2006 (northern Africa) and 22 July 2009 (eastern Asia). The location of the BSRN stations used for each episode is included in Fig. 2. These stations are Florianopolis (FLO) in South America; Carpentas (CAR), Lindenberg (LIN) and Payerne (PAY) in Europe; Tamanrasset (TAM) in northern Africa; and Tateno (TAT) and Xianghe (XIA) in eastern Asia.

For each episode, we create a single domain composed of $200 \times 200$ grid points (Fig. 2) and 50 vertical levels, with a horizontal resolution of $27 \mathrm{~km}$ and the top of the model at $50 \mathrm{hPa}$. All simulations are initialized using the ERA-Interim reanalysis at $0.7^{\circ} \times 0.7^{\circ}$ (Poli et al., 2010) at 18:00 UTC on the day before the date of the eclipse in order to minimize the impact of model spin-up and updating the boundary conditions every $6 \mathrm{~h}$. Other settings related to the model configuration are described in Appendix B because this information is not relevant to the experiments presented here.

Although the new code has an impact on all shortwave parameterizations, we reduce the discussion to Dudhia (Dudhia, 1989) for two reasons: (i) the eclipse modifies the incoming radiation that is the same for all schemes (Sect. 2), and (ii) Dudhia is the simplest shortwave parameterization available in the model and therefore suitable for these initial experiments.

For each case, we run two simulations, one using the default version of the WRF-ARW model (release 3.6.1) used as a "control simulation" and one using the new implementation. In both cases, the cloud interaction within the solar scheme is disabled using the parameter "icloud" in the "namelist.input" file. There are two reasons for disabling the cloud effects. On the one hand, cloud determination is one of the most important sources of error in mesoscale models, and hence they only add noise to the discussion. On the other hand, the main goal of this study is the implementation of Bessel's method, shifting clouds to a secondary role. Moreover, the horizontal resolution used for these experiments cannot produce the desirable cloud granularity to be compared with on-site real time series. Nevertheless, the microphysics scheme is enabled to obtain a more realistic response of the model. In the following sections, the baseline version of the model without the eclipse physics will be referred to as "WRF3.6.1" and the model version with the eclipse algorithm will be called "WRF-eclipse".

The set of results presented in this study is provided with a 1 min time resolution in order to capture the relevant variations during the solar eclipse. Spatially, the nearest grid point is selected to represent each BSRN site.

\section{Results}

This section includes a discussion of the results produced by the simulations described in Sect. 4. The analysis is divided into two parts. First, the skill of the new algorithm in reproducing the impact of the eclipse on the solar radiation received at the ground for different locations and eclipse events will be evaluated (Sect. 5.1). In the second part, we will present an initial discussion about the model response comparing the study cases presented in this paper with the previous results described in Eaton et al. (1997) and Founda et al. (2007), among others (Sect. 5.2).

Before presenting the results of the analysis, three parameters used in the discussion should be defined. The first is the "First Contact Time in Domain" (FCTD), described as 

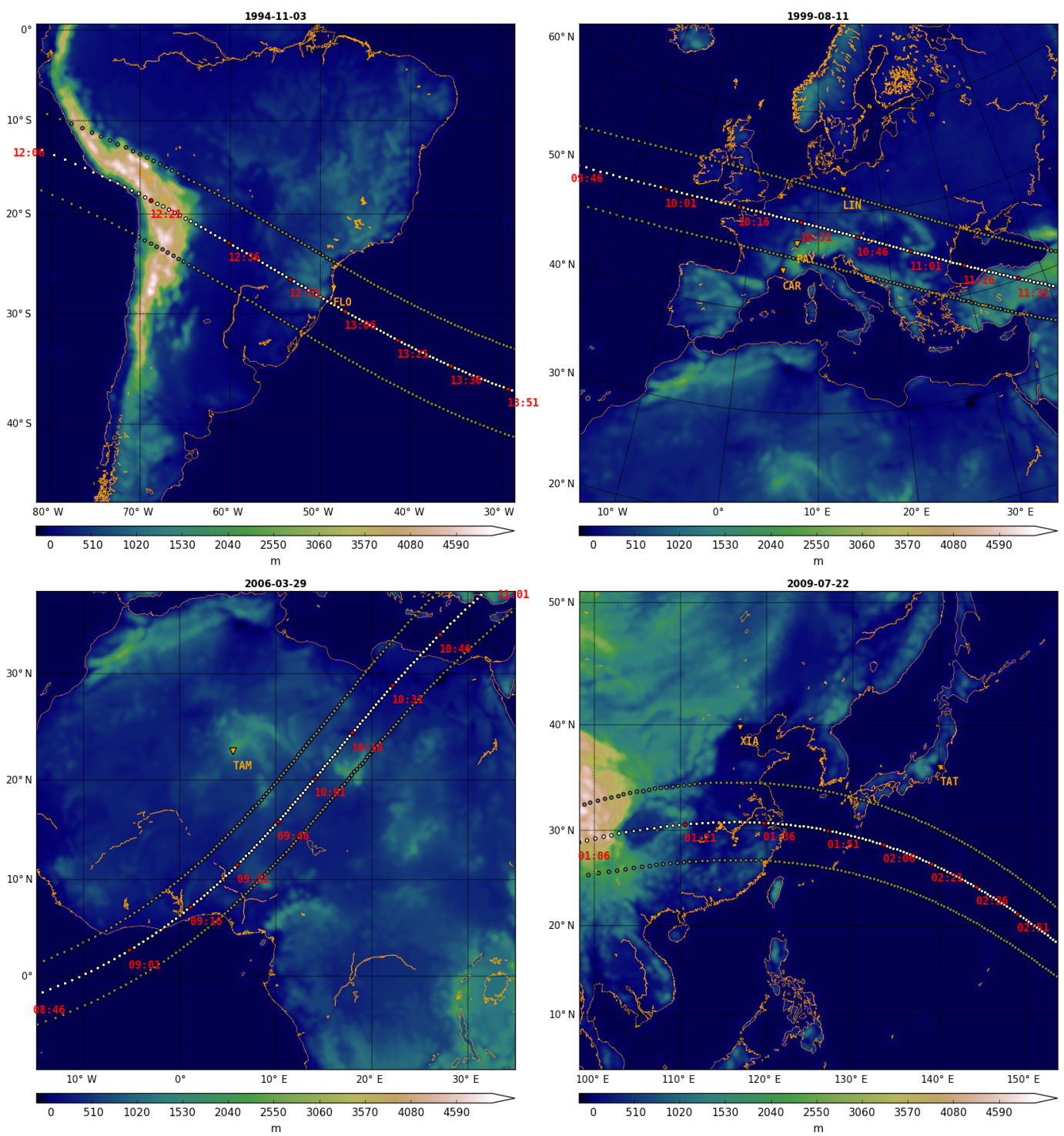

Figure 2. Domains used for each case study. Sites are indicated in orange. The eclipse track is represented by the central gray points. The red points indicate some of the eclipse times in UTC. The dark gray points show the bounds of the $90 \%$ obscuration.

the time stamp in which one node in the domain has an obscurity degree different than zero. From that moment, both simulations are not strictly equal because the incoming radiation in WRF-eclipse has been modified. This variation has a direct impact on the solar heating rate profile as well as on the GHI and consequently on the other meteorological fields through (i) the Euler equations and (ii) the land surface model and surface layer parameterizations (Montornès et al., 2015). Trivially, as FCTD is a property of the episode, the magnitude is the same for all sites in the same domain.

In the case studies presented in this paper, we observe that the FCTD occurs $\sim 1 \mathrm{~h}$ earlier than the first contact of the axis of the eclipse in the domain represented in Fig. 2. The physical reason can be easily interpreted considering the velocity of the axis across the Earth and the radius of the shadow. When the episodes are sorted chronologically (i.e., 3 November 1994, 11 August 1999, 29 March 2006 and 22 July 2009), the FCTD is observed at 11:08, 08:39, 07:41 and 00:06 UTC, respectively (Table 1).

The second term that will be useful for describing the results is the "Maximum Obscuration Time" (MOT), the time in which the obscuration degree reaches the maximum value at each site and thus when the GHI reaches the lowest value under the cloudless-sky assumption. This magnitude is certainly site-dependent.

Finally, the "Last Contact Time in Domain" (LCTD) defines the last time stamp in which some grid point has a degree of obscuration different from 0 . As in the case of the FCTD, this variable depends on the episode, with 15:16, 


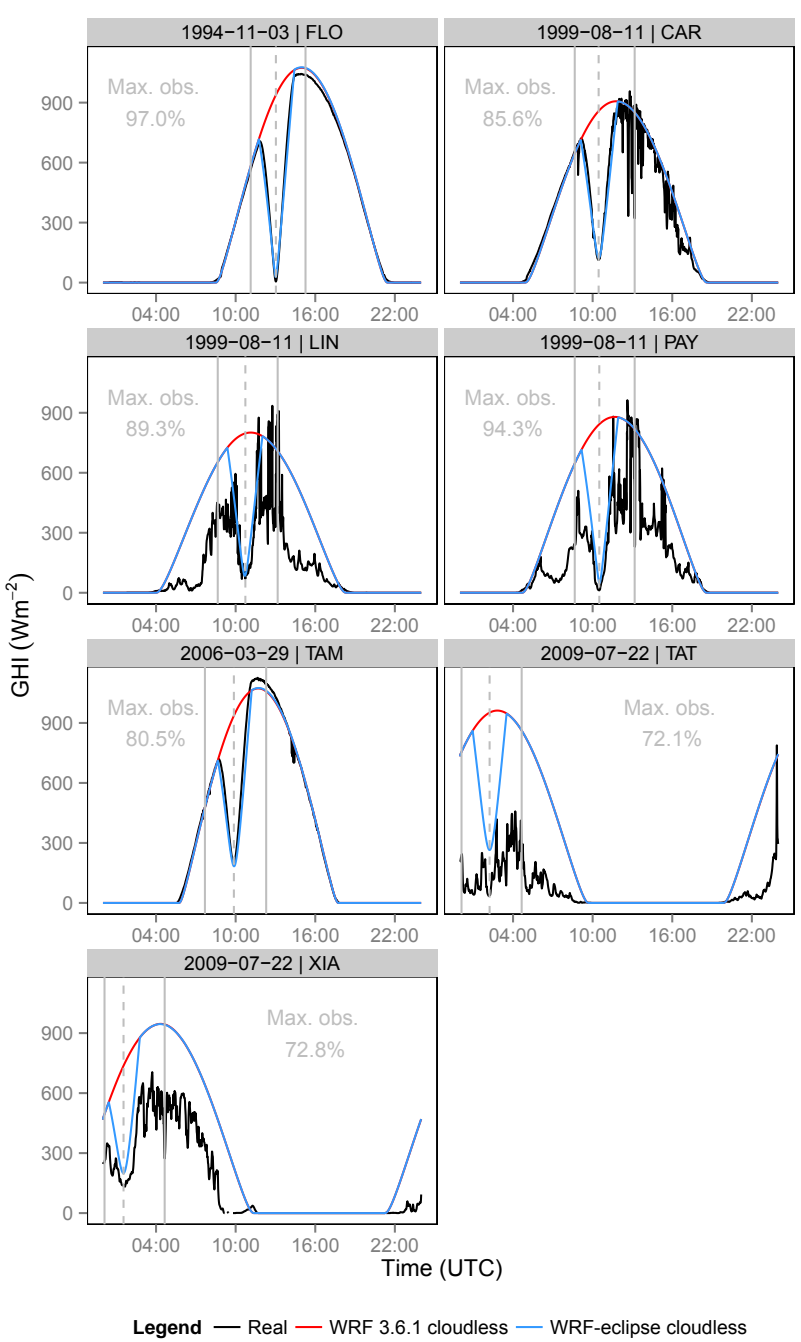

Figure 3. GHI outcomes for each episode and site. Each total solar eclipse event is labeled with the date. Plots show real measurements (in black), the control simulation (red) and the new implementation (blue). The first and second vertical gray solid lines indicate the time of FCTD and LCTD, respectively. The dashed vertical gray line shows the time of maximum obscuration. All results are expressed with 1 min time resolution.

13:10, 12:18 and 04:29 UTC being the times of occurrence (Table 1) for each case study.

\subsection{Global horizontal irradiance}

The study of the GHI shows a similar behavior throughout all analyzed sites (Fig. 3). Before and after the eclipse, the WRF3.6.1 and the WRF-eclipse show identical outcomes, while the first one reproduces the expected cloudless daily pattern during the eclipse and the second decreases abruptly reaching the minimum value at MOT and increasing again later. The reduction in the GHI depends on the eclipse conditions in each place.
Time series for WRF-eclipse are well synchronized with respect to the real data sets. The MOT (Table 1) in FLO is reached at 13:01 UTC. CAR, LIN and PAY show the lowest GHI at 10:28, 10:42 and 10:31 UTC, respectively. TAT and XIA have the lowest GHI at 02:14 and 01:33 UTC. Finally, TAM experiences the MOT at 09:53 UTC, with a delay of $\sim 1$ min with respect to the real measurements. This delay is produced because we are considering the nearest grid point in an equatorial region.

The amplitude of the GHI reduction shows good agreement in those sites showing clear-sky conditions in real measurements (e.g., FLO, TAM), while those sites with clouds (e.g., PAY, TAT) show a tendency to overestimate the GHI because we are not considering the effect of clouds on radiative transfer in these experiments. A similar cloud impact was reported by Founda et al. (2007) when comparing WRFsimulated GHI with real observations during the total solar eclipse of 29 March 2006.

The accuracy is quantified in terms of the bias and the mean absolute error (MAE), defined as

$$
\begin{aligned}
\text { Bias } & =\frac{1}{N} \sum_{i=1}^{N}\left(f_{i}-o_{i}\right), \\
\mathrm{MAE} & =\frac{1}{N} \sum_{i=1}^{N}\left|f_{i}-o_{i}\right|,
\end{aligned}
$$

respectively. The validation period consists of all times with obscuration greater than zero in WRF-eclipse and a solar zenith angle less than $80^{\circ}$ (i.e., it excludes times when the sun is just above the horizon). Hence, $N$ is the number of valid frames, while $f$ and $o$ are the modeled and real values, respectively. These metrics are not normalized with respect to the radiation at TOA as is usually done because this variable is not the same in WRF3.6.1 as in WRF-eclipse.

Sites under cloudless conditions show the highest improvements in MAE (Table 2). In FLO, the use of WRFeclipse represents an improvement of $90 \%$ in the MAE with respect to WRF3.6.1 simulations. TAM shows similar results with a decrease of $77 \%$ in the MAE. Both sites show a high reduction in the bias: in FLO, from 438 to $-34 \mathrm{~W} \mathrm{~m}^{-2}$ and, in TAM, 348 to $-82 \mathrm{~W} \mathrm{~m}^{-2}$. This high underestimation is associated with the near grid point issue mentioned before.

In contrast, lower improvement is observed in cloudy conditions. The best results are detected in CAR with an enhancement of $86 \%$, drifting from a high positive bias of $364 \mathrm{~W} \mathrm{~m}^{-2}$ to a slightly negative one of $-42 \mathrm{~W} \mathrm{~m}^{-2}$. LIN and PAY show a similar improvement of 73 and $71 \%$ in the MAE, respectively. Finally, a minor improvement is observed in the Asian stations, with variations in the MAE of $+50 \%$ in TAT and $+64 \%$ in XIA. The bias drifts from 493 to $176 \mathrm{~W} \mathrm{~m}^{-2}$ in XIA and from 798 to $395 \mathrm{~W} \mathrm{~m}^{-2}$ in TAT. 
Table 1. Overview of the parameters that describe the eclipse episodes at the selected sites. FCTD is the First Contact Time in Domain, LCTD is the Last Contact Time in Domain, MOT is the Maximum Obscuration Time and Obsc refers to the degree of obscuration.

\begin{tabular}{llccccc}
\hline Episode & Region & FCTD (UTC) & LCTD (UTC) & Site & MOT (UTC) & Obsc (\%) \\
\hline 3 Nov 1994 & S. America & $11: 08$ & $15: 16$ & FLO & $13: 01$ & 97.0 \\
\hline 11 Aug 1999 & Europe & \multirow{2}{*}{$08: 39$} & $13: 10$ & CAR & $10: 28$ & 85.6 \\
& & & & LIN & $10: 42$ & 89.3 \\
& & & & PAY & $10: 31$ & 94.3 \\
\hline 29 Mar 2006 & Africa & $07: 41$ & $12: 18$ & TAM & $09: 31$ & 80.5 \\
\hline 22 Jul 2009 & Asia & \multirow{2}{*}{$00: 06$} & $04: 29$ & TAT & $02: 14$ & 72.1 \\
& & & & XIA & $01: 33$ & 72.8 \\
\hline
\end{tabular}

Table 2. Bias and MAE of the GHI forecasts produced by WRF3.6.1 and WRF-eclipse for the period in which the obscuration is greater than zero and the solar zenith angle is less than $80^{\circ}$.

\begin{tabular}{llrrrrrr}
\hline Episode & Region & Site & $\begin{array}{r}\text { Duration } \\
(\mathrm{min})\end{array}$ & $\begin{array}{r}\text { WRF3.6.1 } \\
\text { bias }\left(\mathrm{W} \mathrm{m}^{-2}\right)\end{array}$ & $\begin{array}{r}\text { WRF3.6.1 } \\
\text { MAE }\left(\mathrm{W} \mathrm{m}^{-2}\right)\end{array}$ & $\begin{array}{r}\text { WRF-eclipse } \\
\text { bias }\left(\mathrm{W} \mathrm{m}^{-2}\right)\end{array}$ & $\begin{array}{r}\text { WRF-eclipse } \\
\text { MAE }\left(\mathrm{W} \mathrm{m}^{-2}\right)\end{array}$ \\
\hline 3 Nov 1994 & S. America & FLO & 160 & 438 & 438 & -34 & 44 \\
\hline 11 Aug 1999 & Europe & CAR & 167 & 364 & 364 & -42 & 50 \\
& & LIN & 159 & 478 & 480 & 94 & 130 \\
& & PAY & 166 & 580 & 580 & 164 & 170 \\
\hline 29 Mar 2006 & Africa & TAM & 153 & 348 & 352 & -82 & 82 \\
\hline \multirow{2}{*}{ 22 Jul 2009 } & Asia & TAT & 153 & 798 & 798 & 395 & 395 \\
& & XIA & 140 & 493 & 493 & 176 & 176 \\
\hline
\end{tabular}

\subsection{Response of the WRF-ARW model}

Shortwave schemes have a remarkable role within NWP models and are more significant in cloudy situations than in cloudless ones due to the approximations assumed in the computation of the radiative transfer equation. The selection of one solar parameterization or another produces differences in the heating rate profile as well as in the surface energy balance leading to variations in the other fields due to the high nonlinear relationships between the dynamics and the physics of the model (Montornès et al., 2015).

Including solar eclipses within the mesoscale model is conceptually the same issue. The shadow of the Moon reduces the GHI as viewed in Sect. 5.1 and the heating produced by ozone and water vapor absorption in the stratosphere and troposphere, respectively. As a consequence, the surface energy balance is modified reducing the available energy to be transformed into latent, sensible and ground heat, while the diabatic term in the energy equation decreases significantly, producing changes in the other fields.

The following analyses are focused on the surface variables, in particular, the surface heat fluxes, temperature at $2 \mathrm{~m}$ and wind speed and direction at $10 \mathrm{~m}$. Surface fluxes and temperature are analyzed because they are the most direct response to the GHI perturbations. On the other hand, the surface wind is chosen since it provides an indirect and integrated response to the GHI perturbations because it incorporates pressure gradient changes as well as variations in turbulence (i.e., stability). The discussion is focused on the same BSRN sites analyzed in Sect. 5.1, for consistency. However, the model outcomes are not compared with real measurements because the temporal resolution of the weather variables in the BSRN stations is 3-hourly given that they report these data sets to the SYNOP network and, consequently, they cannot provide the required temporal granularity to analyze the effects of the eclipse over the real atmosphere. Furthermore, the model configuration used in these experiments is not appropriated for a full description of the atmospheric response as can be found in Founda et al. (2007) and others. Consequently, we present a review of the meteorological fields to demonstrate that the model produces reasonable results for the first-order impact. Moreover, a further study of the complex nature of the dynamic response to the eclipseinduced perturbations will be proposed in future works.

The response of the surface fluxes is instantaneous or less than $1 \mathrm{~min}$ as it is observed in Fig. 4 and similar to that observed during a typical sunset and sunrise. Before MOT (Table 1), sensible ( $\mathrm{SH})$ and latent heat $(\mathrm{LH})$ experience a reduction together with an increase in the ground heat $(\mathrm{GH})$. At MOT, these magnitudes reach the maximum difference with 
respect to WRF3.6.1. The range of these deviations varies from one site and episode to the other, as it is related to the degree of obscuration, the time of day and the year (i.e., it is linked to the development of the PBL), land use, soil properties, and weather conditions (i.e., humidity or cloud presence, among others) as indicated by Eaton et al. (1997). The departures in SH between WRF3.6.1 and WRF-eclipse are $\sim 200 \mathrm{~W} \mathrm{~m}^{-2}$, following a similar behavior to that reported by the same study using real measurements. In FLO, CAR and LIN, SH reaches slightly negative values around the MOT. LH experiences larger departures than $\mathrm{SH}$, with differences larger than $\sim 250 \mathrm{~W} \mathrm{~m}^{-2}$ in FLO and CAR. TAM and TAT experience $\mathrm{LH}$ variations lower than $\mathrm{SH}$ due to the local meteorological features. The GH shows a significant increase reaching near-zero values or even slightly positive ones as a response of the $\mathrm{SH}$ decay, as it is highly dependent on the soil features. The largest GH deviations between WRF3.6.1 and WRF-eclipse are produced in TAM because this site is located near the Sahara.

After the MOT (Table 1), surface fluxes in WRF-eclipse tend to return to similar values as in WRF3.6.1. At some sites, such as CAR or XIA, SH experiences greater values than WRF3.6.1 once the solar disk is fully visible again. Other sites with a high dependence on the daily patterns, as at FLO and TAM, show lower SH values.

In this discussion of the fluxes, PAY is an exception. This site experiences a time lag in the response with respect to the MOT with positive heat fluxes deviations (Fig. 4). This pattern is produced because the grid point used for the analyses corresponds to a water body as this site is located near a water area (i.e., Lake Neuchâtel).

Surface fluxes are important because they provide the lower boundary condition for evaluating the vertical transport parameterized within the PBL schemes. Within the model, these physical processes are parameterized in three physical packages: the land surface model (LSM), PBL and surface layer scheme. The LSM approximates those processes occurring at the surface (i.e., surface energy budget, evaporation and soil processes, among others), and it returns $\mathrm{SH}$, $\mathrm{LH}$, terrestrial emission and shortwave reflection to the atmospheric model. The PBL scheme parameterizes the vertical transport of momentum, energy and water vapor between the lower levels of the model and the free atmosphere. Both packages interact through the surface layer parameterization. This physical scheme computes the exchange coefficients and the friction velocity required for calculating $\mathrm{SH}$ and LH within the LSM. Moreover, the surface layer parameterization diagnoses the temperature at $2 \mathrm{~m}$ and wind speed at $10 \mathrm{~m}$ based on the similarity theory equations (Stull, 1988).

Therefore, temperature at $2 \mathrm{~m}$ and wind speed at $10 \mathrm{~m}$ are two interesting fields for understanding the response of the model in an eclipse episode at first order, without considering a full analysis of the PBL, would require a special, dedicated study and which is beyond the scope of this one.

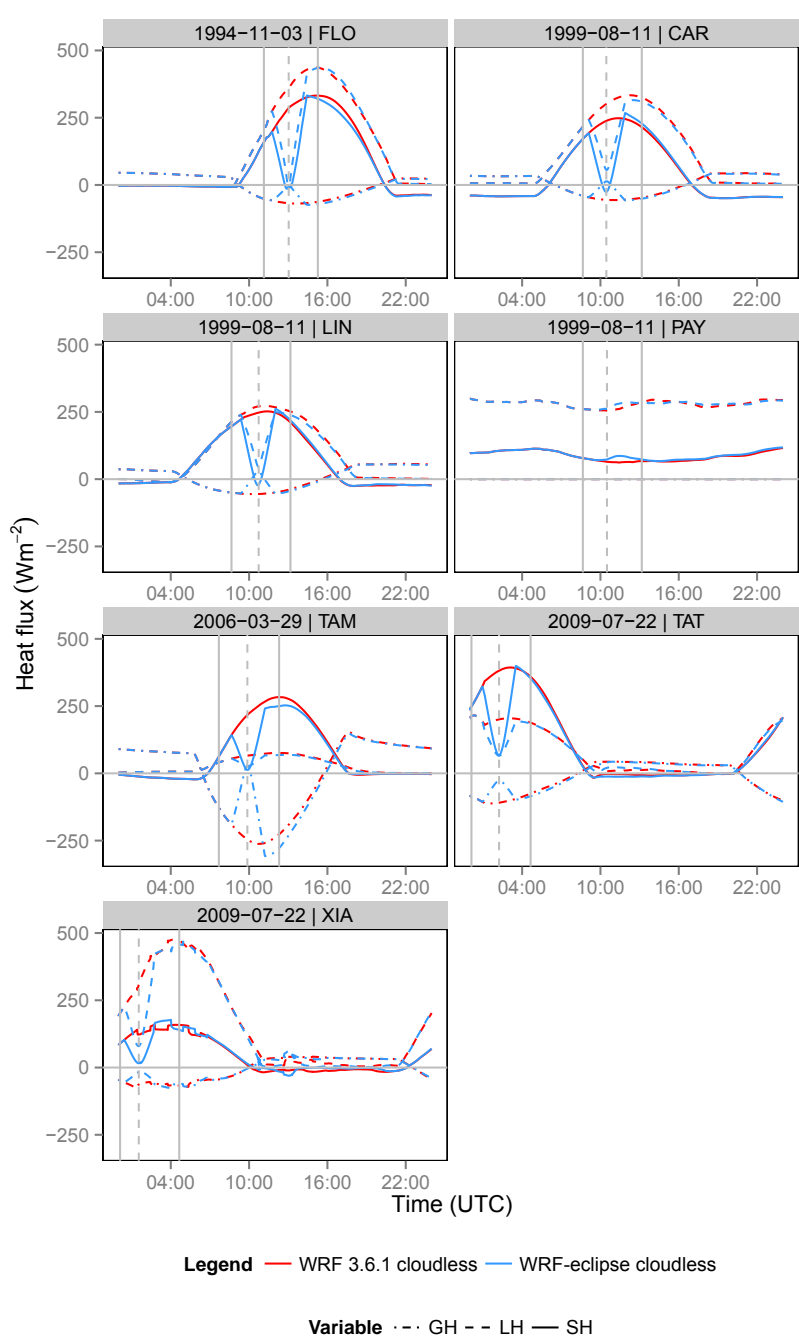

Figure 4. Sensible heat (SH), latent heat (LH) and ground heat (GH) flux outcomes for each episode and site. Each total solar eclipse event is labeled with the date. The GH in PAY is not plotted because the nearest point used is represented by a water body (i.e., GH undefined). Colors indicate the control simulation outcomes (red) and the new implementation (blue). The first and second vertical gray solid lines indicate the time of FCTD and LCTD, respectively. The dashed vertical gray line shows the time of maximum obscuration. All results are expressed with 1 min time resolution.

Variations in the temperature at $2 \mathrm{~m}$ show a delay with respect to the GHI and heat fluxes (Fig. 5). The magnitude and time lag are in agreement to those reported during other solar eclipses using real measurements such as in Fernández et al. (1993b) (between 10 and $30 \mathrm{~min}$ ) or Founda et al. (2007) (between 10 and $15 \mathrm{~min}$ ). FLO shows the greatest variation with $-4.4 \mathrm{~K}, 12 \mathrm{~min}$ after the lowest GHI and SH values (Figs. 3 and 4). CAR, LIN, TAM and TAT experience a similar behavior to each other. In CAR and LIN, the temperature decreases 2.9 and $2.6 \mathrm{~K}, 5$ and $3 \mathrm{~min}$ after the MOT (Table 1), respectively. TAM shows a reduction of $2.1 \mathrm{~K}, 6 \mathrm{~min}$ after the maximum obscuration, while in TAT, the temperature de- 


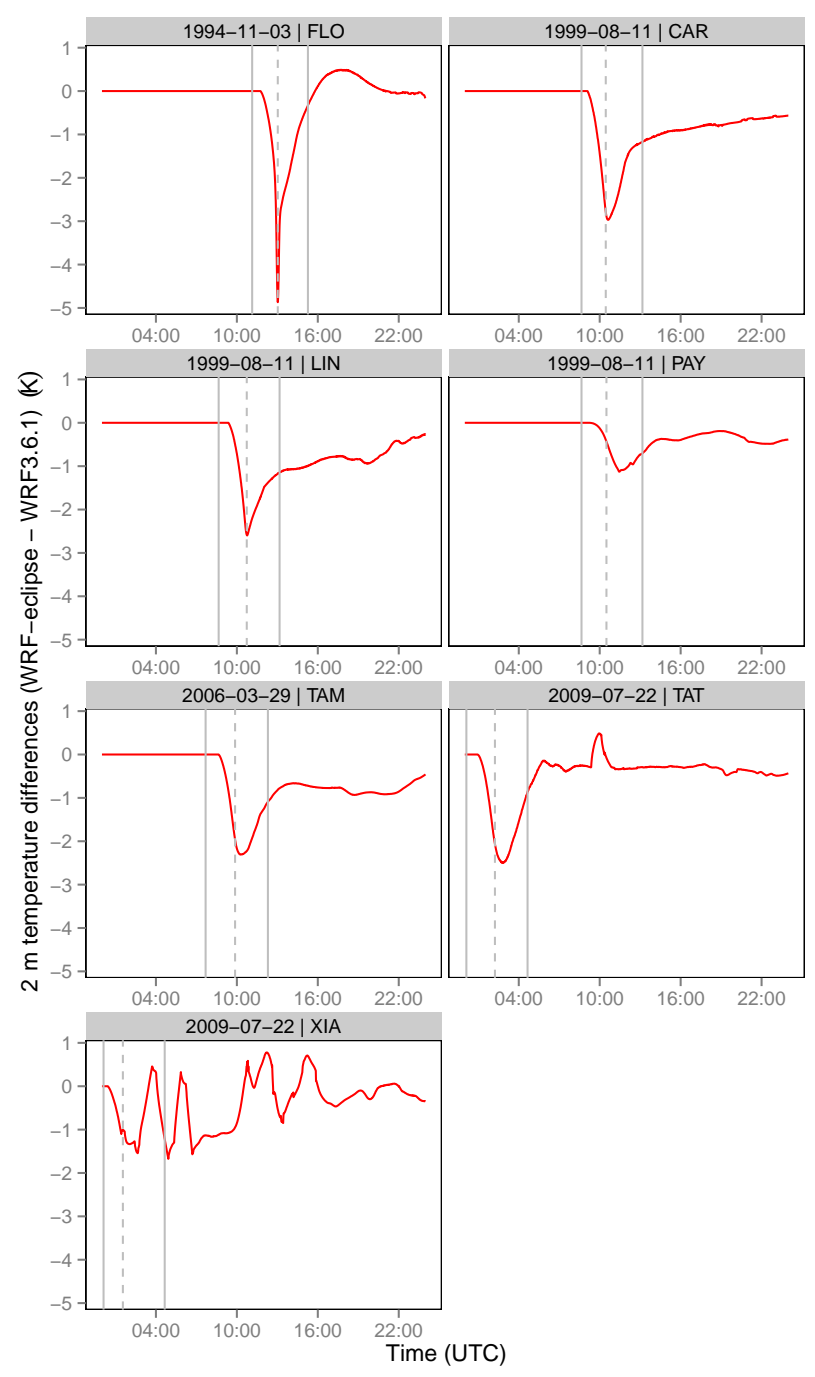

Figure 5. Temperature differences at $2 \mathrm{~m}$ for each episode and site. Each total solar eclipse event is labeled with the date. The first and second vertical gray solid lines indicate the time of FCTD and LCTD, respectively. The dashed vertical gray line shows the time of maximum obscuration. All results are expressed with 1 min time resolution.

cays $2.2 \mathrm{~K}$ with a delay of 4 min. Finally, PAY and XIA show the largest delays with similar variations. In the first case, the minimum value is reached $1 \mathrm{~h}$ and $16 \mathrm{~min}$ after the MOT with an anomaly of $-1.1 \mathrm{~K}$. In the second case, the minimum temperature is shown with a shift of $11 \mathrm{~min}$ and a variation in $-1.2 \mathrm{~K}$. Wu et al. (2011) reported similar temperature decreases during the same solar eclipse over China using the WRF-CHEM module. The amplitude of the temperature decrease in TAM is also in agreement with the WRF simulations in Greece shown by Founda et al. (2007). Nevertheless the local environment and cloudiness determine the actual observed differences.

After the eclipse, all sites tend to return to similar patterns as observed in WRF3.6.1. However, there are some signifi- cant differences. CAR, LIN, PAY and TAM are stations that experience temperatures around 0.5 and $1 \mathrm{~K}$ lower than in WRF3.6.1 outcomes, with PAY being the most conservative case due to the lake effect. TAT shows a positive anomaly between 10:00 and 12:00 UTC, falling quickly to near-zero negative departures. XIA is the site with the highest variation in the temperature, drifting from positive departures $(\sim+0.5 \mathrm{~K})$ to negative ones $(\sim-1 \mathrm{~K}$, even $\sim-1.5 \mathrm{~K})$ during the successive hours, with a tendency to become more stable at the end of the day. These extreme temperature variations are a consequence of the patterns described in the surface fluxes. Finally, FLO experiences positive differences at local midday, drifting to near-zero departures at the local evening. The reason for this positive anomaly is described by the local meteorological conditions. FLO is located on the coast (Fig. 2) and highly influenced by breezes producing a well-defined wind speed pattern (Fig. 6). The eclipse produces a delay in the wind speed daily maximum leading to warmer air at midday with respect to the simulation without the eclipse. Similar results were reported in Subrahamanyam and Anurose (2011), comparing real measurements for an eclipse episode with respect to a control day in a region of India highly influenced by the sea-land breezes.

The response of the wind speed varies from one site to another (Fig. 6) linked to high nonlinear relationships between the model dynamics and physical schemes. The particular variations in wind speed and direction during and after the eclipse are related to the degree of obscuration as well as the local environment and meteorological patterns, as it has been presented in other previous studies (Fernández et al., 1993b; Founda et al., 2007; Wu et al., 2011).

The temporal response in FLO, CAR and LIN is similar to that observed for the temperature (Fig. 5). These sites experience an abrupt reduction in speed, with near-zero deviations some hours after the MOT. CAR and LIN experience a minimum wind speed 5 and $3 \mathrm{~min}$ after the MOT, with -1.8 and $-1.6 \mathrm{~m} \mathrm{~s}^{-1}$, respectively. Both sites show wind speeds above the WRF3.6.1 values (less than $+0.5 \mathrm{~m} \mathrm{~s}^{-1}$ ) after the eclipse linking with the pattern observed in SH (Fig. 4). Variations in wind direction (Fig. 7) are negligible both in CAR and LIN. FLO shows two minima; the first is produced $3 \mathrm{~min}$ after the MOT and the second minimum $\sim 4 \mathrm{~h}$ later, after local midday. Both cases show a wind speed reduction of $\sim 1.5 \mathrm{~m} \mathrm{~s}^{-1}$, with the second one being less important. This observed pattern is a direct consequence of the temperature lag that reduces the sea breeze as it is reflected in wind direction. Between 1 and $2 \mathrm{~h}$ after the MOT, wind direction in WRF-eclipse changes, with a western component (i.e., from land; Fig. 2). During the afternoon and sunset, the thermal lag produced by the solar eclipse disappears and WRF-eclipse returns to the baseline direction.

PAY and TAM show near-zero positive variations at MOT with a positive peak of +0.5 and $+0.8 \mathrm{~m} \mathrm{~s}^{-1}, 40$ and $43 \mathrm{~min}$ after the maximum obscuration (Table 1), respectively. After this peak, wind speed shows a negative minimum, more 


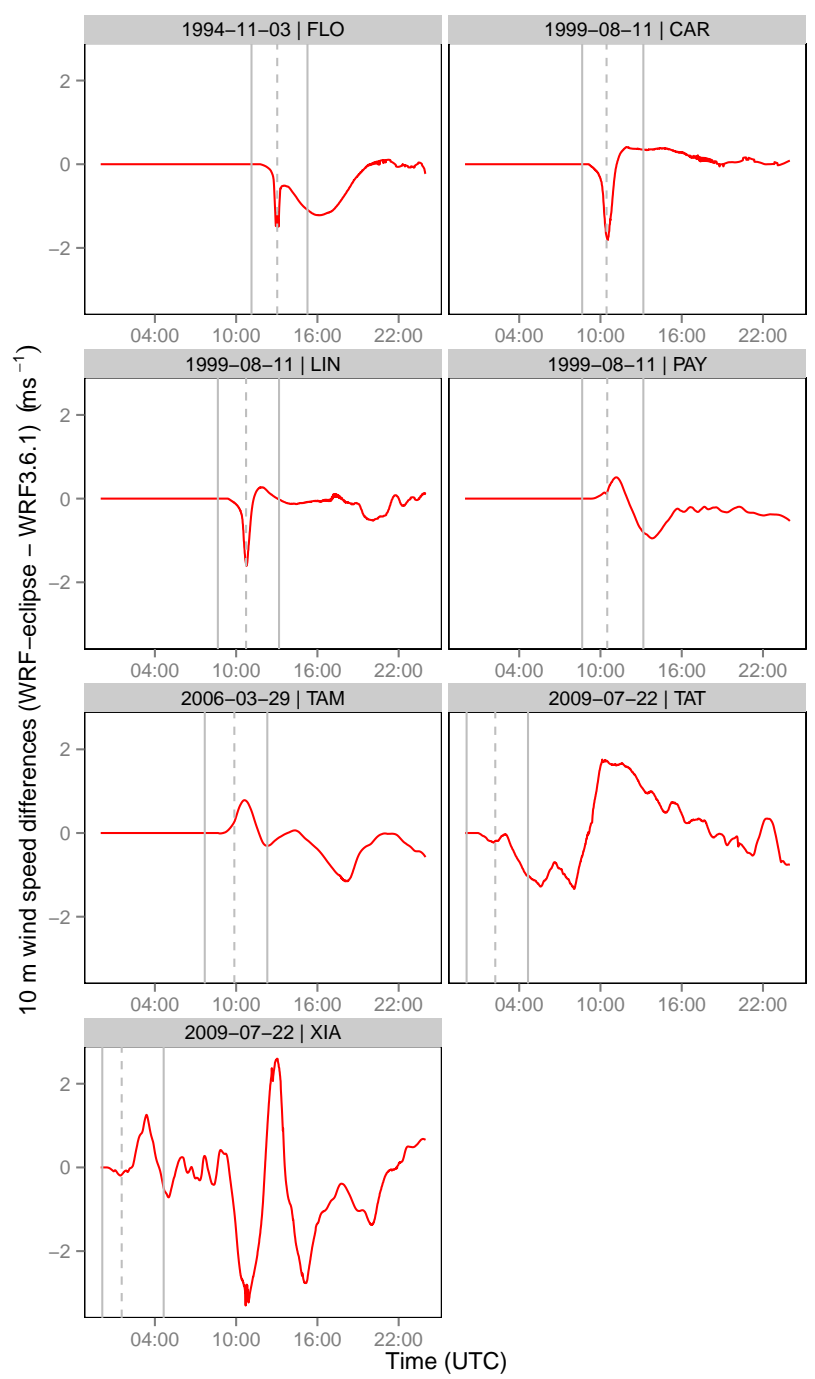

Figure 6. Wind speed differences at $10 \mathrm{~m}$ for each episode and site. The first and second vertical gray solid lines indicate the time of FCTD and LCTD, respectively. The dashed vertical gray line shows the time of maximum obscuration. All results are expressed with 1 min time resolution.

important in PAY than in TAM. During the next hours, PAY experiences slightly lower speeds than in WRF3.6.1, while TAM maintains negative deviations. The reason for this pattern in TAM is the Sahara. As a consequence of the eclipse, the surface of the desert becomes cooler than in WRF3.6.1 producing a weakening of the temperature gradients, and the desert thus experiences lower wind speeds during the afternoon and evening. This behavior is more evident in wind direction (Fig. 7). Just after MOT, WRF-eclipse experiences a significant change with respect to the baseline case.

TAT and XIA show the highest departures between WRFeclipse and WRF3.6.1 compared with the other stations. At the MOT, both sites experience near-zero negative deviations. After the eclipse, the wind differences show a set

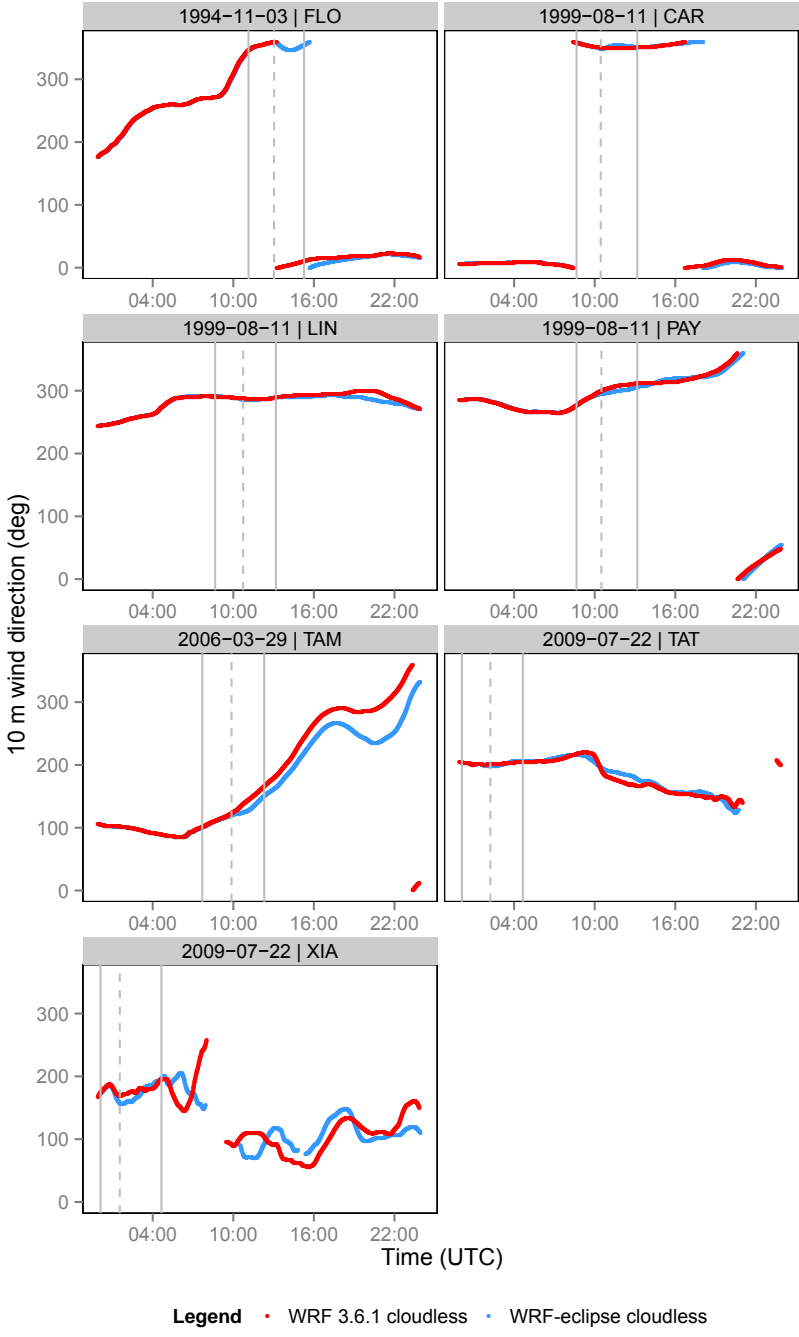

Figure 7. Wind direction at $10 \mathrm{~m}$ for each episode and site. Values for wind speeds below $1 \mathrm{~m} \mathrm{~s}^{-1}$ are not included. The first and second vertical gray solid lines indicate the time of FCTD and LCTD, respectively. The dashed vertical gray line shows the time of maximum obscuration. All results are expressed with $1 \mathrm{~min}$ time resolution.

of positive and negative peaks in time, noisier and larger in XIA (from $\sim-3$ to $\sim 2.5 \mathrm{~m} \mathrm{~s}^{-1}$ ) than in TAT (from $\sim-1$ to $\sim 1.5 \mathrm{~m} \mathrm{~s}^{-1}$ ). TAT shows two well-defined patterns (Fig. 6): one with negative departures before the sunset at $\sim$ 10:00 UTC (Fig. 3) and the other at night with positive departures drifting to near-zero negative values at the end of the day. The reason for this pattern is again the sea breeze and the temperature gradient. TAT is located on the coast of Japan (Fig. 2). In the simulation considering the eclipse, the land reaches lower temperatures, decreasing the gradient with respect to the sea. Consequently, wind speed reaches lower values during daytime hours than in WRF3.6.1. At night, the pattern is reversed when land surface temperatures in WRFeclipse reach lower values faster than in WRF3.6.1, strengthening the gradient with respect to the sea and thus producing 
an increase in the wind. This behavior is reflected in wind direction (Fig. 7). When WRF-eclipse underestimates the wind speed, the wind direction changes to the east. In contrast, when wind speed is overestimated with respect to WRF3.6.1, wind direction changes to the south.

Finally, XIA experiences the most complex patterns in both simulations. Changes in wind direction (Fig. 7) start before the MOT, and they become more important during the hours after the eclipse. The baseline simulation shows a wavy pattern changing from east to southeast. The WRF-eclipse outcome shows a similar behavior but with a shift in time.

The LCTD corresponds to the moment that WRF3.6.1 and WRF-eclipse domains are the same once again in terms of radiative transfer (i.e., respective grid points have the same incoming solar radiation). At this time, the most directly related radiative fields such as the GHI or the surface heat fluxes return to the baseline behavior (Figs. 3 and 4). The surface temperature (Fig. 5) returns to the original pattern more or less quickly depending on the local features and the moment of the day when the eclipse occurs (i.e., effects over the thermal inertia). Wind speed shows more complex patterns due to the high nonlinear relationships between the dynamic fields (Figs. 6 and 7). In general, differences tend to decrease in time in all sites. With the exception of TAM and XIA, all sites return to the original wind direction after some hours of the LCTD, a sign that the model tends to return to the meteorological patterns after dissipating the perturbation introduced by the shadow. The other analyzed meteorological fields need more time for achieving the baseline behavior completely. However, longer simulation horizons are required for reaching a full return to the non-perturbed values.

\section{Conclusions}

This paper describes the implementation of a new package within the WRF-ARW model that includes the effect of the solar eclipses. The presented approach uses Bessel's method and the Five Millennium Catalog of Solar Eclipses provided by NASA for determining the eclipse conditions at each grid point of the domain (Appendix A). Once the position of the Sun and the Moon with respect to the observer (i.e., position within the model domain) are computed, the degree of obscuration is evaluated following geometric relationships. This magnitude is then used for correcting the incoming radiation at each grid point accordingly.

The new algorithm has been validated with respect to NASA's values for the eclipse trajectory covering all total, annular and hybrid eclipses from 1950 to 2050 . This validation shows a good accuracy in the determination of the latitude and longitude for the main requirements of mesoscale model applications. Both variables are computed with a bias lower than $\pm 5 \times 10^{-3}$ degrees (i.e., $\sim 550 \mathrm{~m}$ at the Equator) with a tendency to overestimate the latitude and underestimate the longitude.
In order to demonstrate that this new implementation produces reasonable results for the eclipse-induced perturbations, the code has been tested in real simulations and compared with the default version 3.6.1. The analysis includes four total solar eclipse episodes: 3 November 1994 (South America), 11 August 1999 (Europe), 29 March 2006 (northern Africa) and 22 July 2009 (eastern Asia).

The variations in the solar radiative transfer due to the eclipse produce two impacts on the model: one in the GHI and the other on the heating rate profile. The first one has a contribution in the surface energy budget parameterized in the LSM, while the second one modifies the diabatic term in the energy equation and thus in Euler equations.

The new GHI shows good agreement with respect to the real measurements. The modeled solar eclipse is well synchronized with the reality at all sites. The modeled eclipseinduced GHI perturbations agree very well with the measured perturbations at sites with observed cloudless skies (e.g., TAM, FLO) but the agreement is not as good in observed cloudy scenarios (e.g., TAT, PAY) because the effects of clouds are not included in these experiments. A similar cloud impact was reported by Founda et al. (2007) when comparing WRF-simulated GHI with real observations during the total solar eclipse of 29 March 2006.

The reduction in the GHI leads to instant changes in the $\mathrm{SH}, \mathrm{LH}$ and GH fluxes. The response of these fields varies as a function of the degree of obscuration and the land use. As a consequence, the PBL experiences changes that are represented by the temperature at $2 \mathrm{~m}$ and wind speed at $10 \mathrm{~m}$. In general, both fields experience an abrupt reduction, while the solar disk is hidden by the Moon, and this reduction is faster in temperature than in wind speed. After the eclipse, all analyzed sites tend to return to values similar to those in WRF3.6.1.

The response in the temperature at $2 \mathrm{~m}$ varies from $\sim-1$ to $\sim-3 \mathrm{~K}$, with a time lag between $\sim 5$ and $\sim 15 \mathrm{~min}$ after the maximum obscuration (Table 1). In places over water bodies this delay is larger, requiring more than $1 \mathrm{~h}$ due to the thermal inertia of water. On the other hand, the response of wind speed and direction at $10 \mathrm{~m}$ are strongly influenced by the temperature changes. Thus, the solar eclipse has a large impact on those sites near the coast due to the effects on the land-sea breeze.

In conclusion, the presented implementation proves to be an interesting tool for the solar industry and for research applications. On the one hand, the study provides an integrated modeling approach for solar power generation forecasting applications that can be an interesting tool after the episodes which occurred in the USA (23 October 2014) and Europe (20 March 2015).

On the other hand, solar eclipses induce real perturbations that provide a good opportunity for a better understanding of the feedback between the atmospheric components as well as the degree of realism in the relationships between physical parameterizations in NWP models. The presented study 
has laid the groundwork for subsequent studies of the complex nature of the dynamic response to the eclipse-induced perturbations with a large number of case studies. Examples of possible future studies are a full validation of surface and vertical fields using ground-based stations and soundings, a full study of the gravity waves induced by changes in the heating rate profile in the stratosphere, a description of the modification in the local-scale meteorological patterns or a study of the PBL response. 


\section{Appendix A}

Physically, a solar eclipse occurs when the lunar and solar centers are distant from one another by an arc in the celestial sphere equal to the sum of their radii (Buchanan, 1904). For many centuries, earlier astronomers tried to determine the occurrence of a solar eclipse based on the movement of both celestial bodies in the sky. Although they made highaccuracy predictions, the method was not efficient because of the laborious computations being only valid for a given place. In the 18th century, after Kepler's laws and the Principia of Sir Isaac Newton, astronomers such as Edmund Halley made different eclipse predictions based on the Earth and Moon orbits, but the mathematical treatment was highly complex due to the Earth and Moon's respective movements (i.e., translation, rotation, nutation) around the Sun.

At the beginning of the 19th century, Friedrich Wilhelm Bessel developed a new method providing a high-level mathematical simplification independent of the observer. In fact, the approach is more general and it is valid for predicting the place and time for observing all celestial phenomena as occultations and eclipses. This method is still being used in the computer algorithms used for solar eclipse predictions (e.g., NASA).

The method was widely detailed in books and manuals such as Chauvenet (1871). However, we include a brief description of Bessel's method in this appendix in order to contextualize the implementation within the WRF-ARW model described in Sect. 2.

The main idea of this approach is to reduce the problem to a single plane which passes through the Earth's center and which is perpendicular to the axis of the Moon's shadow. This plane is named fundamental plane.

Before following with the description, let us define a Cartesian coordinate system in $\mathbb{R}^{3}$ in which the $X$ and $Y$ axes are constructed on the fundamental plane, with the origin at the Earth's center. In this new reference system, let us assume that the positive $X$ axis runs in the eastern direction and the $Y$ axis runs in the northern direction. By construction, the $Z$ axis is normal to the fundamental plane and parallel to the axis of the shadow. This new system is more useful than the one located at the Earth's surface because we can define a set of magnitudes relative to the fundamental plane that are independent of the observer.

These variables are the coordinates $x$ and $y$ of the point where the shadow axis crosses the fundamental plane, the direction of the shadow axis in the celestial sphere described by the declination $d$ and the ephemeride solar angle $\mu$, the radii of the penumbral and umbral shadows $l_{1}$ and $l_{2}$ in the fundamental plane, and the angle that the penumbral $\alpha_{1}$ and umbral $\alpha_{2}$ shadow cones make with the shadow axis defined by $f_{1}=\tan \alpha_{1}$ and $f_{2}=\tan \alpha_{2}$. This set of variables is named Besselian elements, and they only depend on time in the $X Y Z$ system. Therefore, the Besselian elements can be computed before an eclipse and used to determine the episode features as the trajectory of the shadow or the visibility in any place around the Earth.

For the particular case of the solar eclipses, NASA supplies two catalogs: the Five Millennium Catalog of Solar Eclipses (Espenak and Meeus, 2008) that contains all episodes since $2000 \mathrm{BCE}$ to $3000 \mathrm{CE}$ and the Ten Millennium Catalog of Long Solar Eclipses (Espenak and Meeus, 2009) with a period from $4000 \mathrm{BCE}$ to $6000 \mathrm{CE}$.

In these catalogs, NASA provides for each eclipse a reference time $t_{0}$ in the Terrestrial Dynamical Time (TDT) reference system and a set of polynomial coefficients to compute the Besselian elements valid in a $6 \mathrm{~h}$ period centered on $t_{0}$ (i.e., $t_{0} \pm 3$ ).

For a given eclipse and time $t_{1}$ in TDT, the Besselian elements are evaluated as

$$
\begin{aligned}
& x=x_{0}+x_{1} t+x_{2} t^{2}+x_{3} t^{3}, \\
& y=y_{0}+y_{1} t+y_{2} t^{2}+y_{3} t^{3}, \\
& d=d_{0}+d_{1} t+d_{2} t^{2}, \\
& \mu=\mu_{0}+\mu_{1} t+\mu_{2} t^{2}, \\
& l_{i}=l_{i, 0}+l_{i, 1} t+l_{i, 2} t^{2},
\end{aligned}
$$

with $i=1,2$ (penumbra and umbra) and where $t=t_{1}-t_{0}$ in TDT. Note that the cone angles $f_{1}$ and $f_{2}$ are assumed to be constant during all the eclipse (i.e., $t_{0} \pm 3 \mathrm{~h}$ ). By construction, the penumbra shadow radius in the fundamental plane $l_{1}$ is always defined as a positive value, while the umbra shadow radius $l_{2}$ is defined as positive for annularity and negative for totality.

Internally, the WRF-ARW model considers the time in the Coordinated Universal Time (UTC) system. Therefore, before computing the Besselian elements, this time should be converted to TDT. This conversion is performed using a variable that astronomers call "delta- $T$ " or, hereinafter, $\Delta t$. Conceptually, this parameter is a correction to time due to the differences in the Earth rotation produced by the angular momentum transferred from Earth to the Moon by the tidal friction. This variable is also provided in the catalogs for eclipses.

Thus, $t$ is computed as

$t=t_{1}^{\mathrm{TDT}}-t_{0}^{\mathrm{TDT}}=\left(t_{1}^{\mathrm{UTC}}-\Delta t\right)-t_{0}^{\mathrm{TDT}}$.

The key point in order to implement the eclipses in an NWP model is the determination of the degree of obscuration $D$ of the solar disk at each grid point of the domain (Sect. 2). Each grid point is characterized by two geographical coordinates given by the latitude $\phi$ and longitude $\lambda$. Therefore, first of all, we need to transform this pair of coordinates into the reference system $X Y Z$.

Nevertheless, we need to introduce a couple of corrections to the geographical coordinates provided by the atmospheric 
model. As the real Earth is an ellipsoid, we need to correct the geographical latitude with the eccentricity, $\epsilon$ as

$\tan \phi_{1}=\tan \phi \sqrt{1-\epsilon^{2}}$,

where $\epsilon$ is taken as 0.0818192 from Meeus (1991).

On the other hand, the geographic longitude $\lambda$, which refers to the Greenwich meridian, must be transformed to the ephemeride longitude $\lambda_{1}$ by applying the correction

$\lambda_{1}=\lambda+1.002738 \frac{15 \Delta t}{3600}$.

Then, if $\xi, \eta$ and $\zeta$ are the coordinates of the observer in the $X Y Z$ reference system, we can express the coordinate transform as

$\xi=\cos \phi_{1} \sin H$,

$\eta_{1}=\frac{\eta}{\rho_{1}}=\sin \phi_{1} \cos d_{1}-\cos \phi_{1} \sin d_{1} \cos H$,

$\zeta_{1}=\frac{\zeta}{\rho_{2}}=\sin \phi_{1} \sin d_{2}-\cos \phi_{1} \cos d_{2} \cos H$,

where $H$ is the hour angle in the observation place (i.e., grid point) defined as

$H=\mu-\lambda_{1}$

and $\rho_{1}, d_{1}, \rho_{2}$ are $d_{2}$ are a set of variables given by the following relationships

$\rho_{1} \sin d_{1}=\sin d$,

$\rho_{1} \cos d_{1}=\cos d \sqrt{1-\epsilon^{2}}$,

$\rho_{2} \sin d_{2}=\sin d \sqrt{1-\epsilon^{2}}$

$\rho_{2} \cos d_{2}=\cos d$.

In the fundamental plane, the eclipse conditions of a grid point $(\xi, \eta)$ are determined by the distance $\Delta$ to the shadow axis $(x, y)$. Thus,

$\Delta^{2}=(x-\xi)^{2}+\left(y_{1}-\eta_{1}\right)^{2}$.

Equation (A17) defines a circle centered on the shadow axis and concentric to the circles defined by the penumbra and umbra radii, $l_{1}$ and $l_{2}$. Here $y_{1}$ is a correction to $y$ evaluated as

$y_{1}=\frac{y}{\rho_{1}}$.

Typically, the observer will be in a plane parallel to the fundamental plane (i.e., $\zeta \neq 0$ ), named the "observer's plane". As the shadow produced by the Moon is a cone, we need to project the penumbra and umbra radii from the fundamental to the observer's plane (Fig. A1). Based on trigonometric relationships, we can demonstrate that the penumbra $L_{1}$ and umbra $L_{2}$ radii in the observer's plane are given by

$L_{i}=l_{i}-\zeta_{1} f_{i}$,

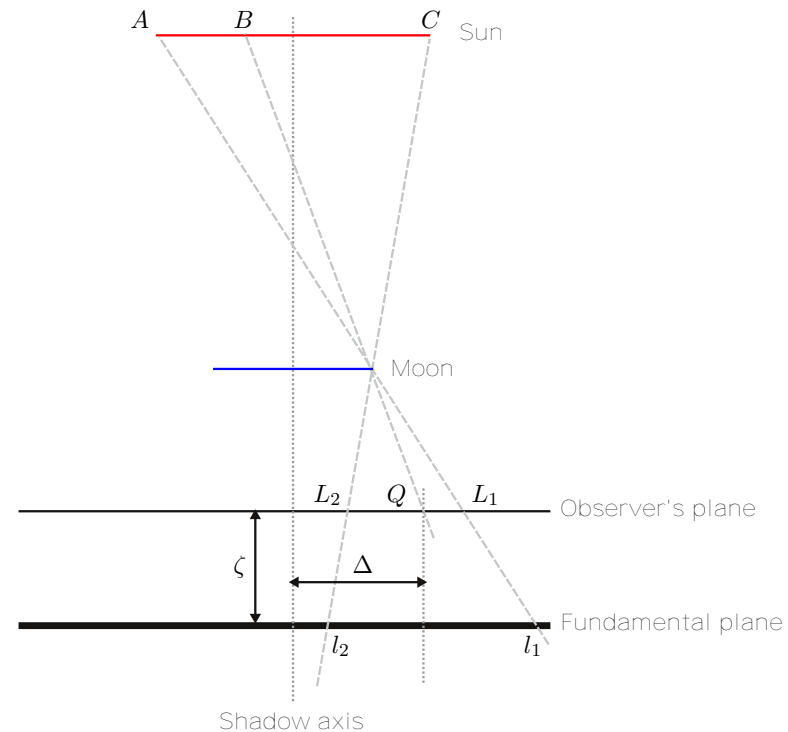

Figure A1. Scheme showing the geometric relationships for determining the degree of obscuration, $D$, in a total solar eclipse. A similar scheme can be drawn in an annular eclipse.

with $i=1,2$.

Therefore, from Eqs. (A17) and (A19), we can define three regions determining the eclipse conditions at the observer's plane. First, when

$L_{1}<\Delta$,

the grid point is located out of the shadow and hence the eclipse is not observable. Second, when

$L_{1} \geq \Delta>\left|L_{2}\right|$,

the observer is within the penumbra region. And finally, if

$\left|L_{2}\right| \geq \Delta \geq 0$

then the node is inside the umbra region.

Therefore, from these ideas along with geometric relationships, we can determine the degree of obscuration $D$ of the solar disk. Formally, we define $D$ as the part of the solar disk that is hidden by the Moon. Let us assume an observer located at a point $Q$ inside the penumbra region with a distance $\Delta$ with respect to the axis of the shadow (Fig. A1). In a situation without eclipse, the observer $Q$ measures the total length of the solar disk as AC. However, during an eclipse, the lunar disk intercepts some of the solar beams and consequently, a part, $\mathrm{AB}$, of the solar disk is not visible from $Q$. Then, the $D$ can be defined mathematically as the ratio between distances $\mathrm{AB}$ and $\mathrm{AC}$ as

$D=\frac{\mathrm{AB}}{\mathrm{AC}}$.

Note that if the observer moves outward to the penumbra region, the length $\mathrm{AB}$ will be shorter until reaching a point 
in which $\Delta$ becomes $L_{1}$ and the distance $\mathrm{AB}$ is zero. At this point, solar and lunar limbs are in contact but the solar disk is not hidden (i.e., $D=0$ ). On the other hand, when $\Delta$ becomes less than $\left|L_{2}\right|$, the solar disk is completely hidden by the Moon (i.e., solar beams cannot reach the Earth's surface). In this case, the observer is inside the umbra region and will experience the annularity or totality depending on the sign of $L_{2}$.

This description can be quantified expressing $D$ as

$$
D=\frac{Q L_{1}}{L_{1} L_{2}} .
$$

This equation can be approximated as

$$
D=\frac{L_{1}-\Delta}{L_{1}+L_{2}} .
$$

Note that by construction, annular solar eclipses always have a denominator greater than the numerator. Therefore, $D$ is always lower than the unity. In contrast, total solar eclipses reach the unity when $\Delta=\left|L_{2}\right|$ because $L_{2}$ is a negative value.

In the validation of the algorithm discussed in Sect. 3, the eclipse trajectories are evaluated with respect to NASA's values. Thus, we need to determine the geographic coordinates of the axis of the shadow over the Earth's surface.

By construction, all points with $\Delta=0$ are in the axis of the shadow, or in other words, all points with $\xi=x$ and $\eta_{1}=y_{1}$ are in the eclipse trajectory.

Therefore, the problem is reduced to find the pairs of geographical coordinates $\phi$ and $\lambda$ for each $x$ and $y_{1}$. Mathematically, we can write the following equation system

$$
\begin{aligned}
\sin \beta \sin \gamma & =x, \\
\sin \beta \cos \gamma & =y_{1}, \\
c \sin C & =y_{1}, \\
c \cos C & =\cos \beta, \\
\cos \phi_{1} \sin H & =x, \\
\cos \phi_{1} \cos H & =c \cos \left(C+d_{1}\right), \\
\sin \phi_{1} & =c \sin \left(C+d_{1}\right), \\
\tan \phi & =\frac{\tan \phi_{1}}{\sqrt{1-\epsilon^{2}}}, \\
\lambda_{1} & =\mu-H, \\
\lambda & =\lambda_{1}-1.002738 \frac{15 \Delta t}{3600} .
\end{aligned}
$$

This system of equations has a solution when $|\sin \beta|<0$. This occurs when the shadow axis passes through the Earth's surface (i.e., total, annular and hybrid eclipses). In the partial eclipses and some total and annular polar eclipses $|\sin \beta|>$ 0 , and the trajectory is not defined over the Earth's surface.

\section{Appendix B}

In this appendix, we detail the model configuration used for the experiments as an extension of the description presented in Sect. 4. All domains are composed of $200 \times 200$ points with a resolution of $27 \mathrm{~km}$ and 50 vertical levels automatically distributed by the model. The top of the model is set at $50 \mathrm{hPa}$. The Euler equations are integrated by using an adaptive time step. The first guess is set to $30 \mathrm{~s}$ with a target in the CFL condition of 1.2. The time step cannot increase more than $60 \mathrm{~s}$ because this is the output frequency for the history file.

The projection used at each domain depends on the BSRN location. In the eclipses of 3 November 1994 (South America), 29 March 2006 (Africa) and 22 July 2009 (Asia), we set a Mercator geographical projection, while for the eclipse of 11 August 1999 (Europe), we used a Lambert Conic Conformal geographical projection tangent to the standard latitude.

All simulations use the same physical schemes. For microphysics the WRF Single-moment 5-class Scheme (Hong et al., 2004) is used. Radiative processes are parameterized using the Rapid Radiative Transfer Model for GCMs (RRTMG; Iacono et al., 2008) for the terrestrial part of the spectrum and Dudhia (1989) for the solar part as indicated in Sect. 4. Radiative transfer codes are called every minute. Surface processes are modeled with the Unified Noah Land Surface Model (Tewari et al., 2004). The vertical transport is parameterized in terms of the Yonsei University Scheme for the PBL based on Hong et al. (2006), called at every time step. The interaction between the LSM and the PBL is performed by the Fifth-Generation PSU/NCAR Mesoscale Model (MM5) Similarity Scheme (Paulson, 1970; Dyer and Hicks, 1970; Webb, 1970; Beljaars, 1995; Zhang and Anthes, 1982). As we set a coarse horizontal resolution, the KainFritsch scheme (Kain, 2004) option for cumulus is also enabled. Higher-order physical parameterizations such as lake surface schemes are not used in the current study. Regarding the dynamics, default settings are used in all experiments. 
Acknowledgements. The ECMWF ERA-Interim data used in this study were obtained from the ECMWF data server. Eclipse Predictions are by Fred Espenak (NASA GSFC).

BSRN investigators and site scientist are thanked for maintaining and improving this data network for the entire scientific community. Imma Torras is thanked for her collaboration preparing the Besselian elements for use within the WRF-ARW model.

We appreciate all suggestions and comments from the anonymous referees and the editor that have without doubt improved the work presented in this paper.

Edited by: P. Monks

\section{References}

Abram, J. P., Creasey, D. J., Heard, D. E., Lee, J. D., and Pilling, M. J.: Hydroxyl radical and ozone measurements in England during the solar eclipse of 11 August 1999, Geophys. Res. Lett., 27, 3437-3440, doi:10.1029/2000GL012164, 2000.

Adrian, G. and Fiedler, F.: Simulation of unstationary wind and temperature fields over complex terrain and comparison with observations, Contribution Atmos. Phys., 64, 27-48, 1991.

Altadill, D., Solé, J. G., and Apostolov, E. M.: Vertical structure of a gravity wave like oscillation in the ionosphere generated by the solar eclipse of August 11, 1999, J. Geophys. Res.-Space, 106, 21419-21428, doi:10.1029/2001JA900069, 2001.

Anderson, J.: Meteorological changes during a solar eclipse, Weather, 54, 207-215, doi:10.1002/j.14778696.1999.tb06465.x, 1999.

Anderson, R. C., Keefer, D. R., and Myers, O. E.: Atmospheric Pressure and Temperature Changes During the 7 March 1970 Solar Eclipse, J. Atmos. Sci., 29, 583-587, doi:10.1175/15200469(1972)029<0583:APATCD>2.0.CO;2, 1972.

Anfossi, D., Schayes, G., Degrazia, G., and Goulart, A.: Atmospheric Turbulence Decay During the Solar Total Eclipse of 11 August 1999, Bound.-Lay. Meteorol., 111, 301-311, doi:10.1023/B:BOUN.0000016491.28111.43, 2004.

Antonia, R. A., Chambers, A. J., Phong-Anant, D., Rajagopalan, S., and Sreenivasan, K. R.: Response of atmospheric surface layer turbulence to a partial solar eclipse, J. Geophys. Res.-Oceans, 84, 1689-1692, doi:10.1029/JC084iC04p01689, 1979.

Aplin, K. L. and Harrison, R. G.: Meteorological effects of the eclipse of 11 August 1999 in cloudy and clear conditions, P. R. Soc. Lond. A Mat., 459, 353-371, doi:10.1098/rspa.2002.1042, 2003.

Arritt, R. W.: Numerical modelling of the offshore extent of sea breezes, Q. J. Roy. Meteor. Soc., 115, 547-570, doi:10.1002/qj.49711548707, 1989.

Ballard, H. N., Valenzuela, R., Izquierdo, M., Randhawa, J. S., Morla, R., and Bettle, J. F.: Solar eclipse: Temperature, wind, and ozone in the stratosphere, J. Geophys. Res., 74, 711-712, doi:10.1029/JB074i002p00711, 1969.

Beljaars, A. C. M.: The parametrization of surface fluxes in largescale models under free convection, Q. J. Roy. Meteor. Soc., 121, 255-270, doi:10.1002/qj.49712152203, 1995.
Bojkov, R. D.: The ozone variations during the solar eclipse of 20 May 1966, Tellus A, 20, 417-421, doi:10.3402/tellusa.v20i3.10020, 1968.

Buchanan, R.: The mathematical theory of eclipses according to Chauvenet's transformation of Bessel's method explained and illustrated, to which are appended Transits of Mercury and Venus and Occultations of fixed stars, J.B. Lippincott company, Philadelphia, 1904.

Chauvenet, W.: A Manual of Spherical and Practical Astronomy, J. B. Lippincott, 1871.

Chimonas, G.: Lamb waves generated by the 1970 solar eclipse, Planet. Space Sci., 21, 1843-1854, doi:10.1016/00320633(73)90115-3, 1973.

Chimonas, G. and Hines, C. O.: Atmospheric gravity waves induced by a solar eclipse, J. Geophys. Res., 75, 875-875, doi:10.1029/JA075i004p00875, 1970.

Chimonas, G. and Hines, C. O.: Atmospheric gravity waves induced by a solar eclipse, 2, J. Geophys. Res., 76, 7003-7005, doi:10.1029/JA076i028p07003, 1971.

Davis, M. J. and Da Rosa, A. V.: Possible Detection of Atmospheric Gravity Waves generated by the Solar Eclipse, Nature, 226, 1123-1123, doi:10.1038/2261123a0, 1970.

Dudhia, J.: Numerical Study of Convection Observed during the Winter Monsoon Experiment Using a Mesoscale Two-Dimensional Model, J. Atmos. Sci., 46, 3077-3107, doi:10.1175/1520-0469(1989)046<3077:NSOCOD>2.0.CO;2, 1989.

Dyer, A. J. and Hicks, B. B.: Flux-gradient relationships in the constant flux layer, Q. J. Roy. Meteor. Soc., 96, 715-721, doi:10.1002/qj.49709641012, 1970.

Eaton, F. D., Hines, J. R., Hatch, W. H., Cionco, R. M., Byers, J., Garvey, D., and Miller, D. R.: SOLAR ECLIPSE EFFECTS OBSERVED IN THE PLANETARY BOUNDARY LAYER OVER A DESERT, Bound.-Lay. Meteorol., 83, 331346, doi:10.1023/A:1000219210055, 1997.

Eckermann, S. D., Broutman, D., Stollberg, M. T., Ma, J., McCormack, J. P., and Hogan, T. F.: Atmospheric effects of the total solar eclipse of 4 December 2002 simulated with a high-altitude global model, J. Geophys. Res., 112, D14105, doi:10.1029/2006JD007880, 2007.

Espenak, F. and Meeus, J.: Five Millennium Catalog of Solar Eclipses: -1999 to +3000 (2000 BCE to $3000 \mathrm{CE}$ ), Tech. rep., NASA, 2008.

Espenak, F. and Meeus, J.: Ten Millennium Catalog of Long Solar Eclipses: -3999 to +6000 (4000 BCE to $6000 \mathrm{CE}$ ), Tech. rep., NASA, 2009.

Fernández, W., Castro, V., and Hidalgo, H.: Air temperature and wind changes in Costa Rica during the total solar eclipse of July 11, 1991, Earth Moon Planets, 63, 133-147, doi:10.1007/BF00575102, 1993a.

Fernández, W., Castro, V., Wright, J., Hidalgo, H., and Sáenz, A.: Changes in solar irradiance and atmospheric turbidity in Costa Rica during the total solar eclipse of July 11, 1991, Earth Moon Planets, 63, 119-132, doi:10.1007/BF00575101, 1993b. 
Fernández, W., Hidalgo, H., Coronel, G., and Morales, E.: Changes in meteorological variables in Coronel Oviedo, Paraguay, during the total solar eclipse of 3 November 1994, Earth Moon Planets, 74, 49-59, doi:10.1007/BF00118721, 1996.

Founda, D., Melas, D., Lykoudis, S., Lisaridis, I., Gerasopoulos, E., Kouvarakis, G., Petrakis, M., and Zerefos, C.: The effect of the total solar eclipse of 29 March 2006 on meteorological variables in Greece, Atmos. Chem. Phys., 7, 5543-5553, doi:10.5194/acp7-5543-2007, 2007.

Fritts, D. C. and Luo, Z.: Gravity wave forcing in the middle atmosphere due to reduced ozone heating during a solar eclipse, J. Geophys. Res., 98, 3011, doi:10.1029/92JD02391, 1993.

Gerasopoulos, E., Zerefos, C. S., Tsagouri, I., Founda, D., Amiridis, V., Bais, A. F., Belehaki, A., Christou, N., Economou, G., Kanakidou, M., Karamanos, A., Petrakis, M., and Zanis, P.: The total solar eclipse of March 2006: overview, Atmos. Chem. Phys., 8, 5205-5220, doi:10.5194/acp-8-5205-2008, 2008.

Gross, P. and Hense, A.: Effects of a Total Solar Eclipse on the Mesoscale Atmospheric Circulation over Europe A Model Experiment, Meteorol. Atmos. Phys., 71, 229-242, doi:10.1007/s007030050057, 1999.

Hanna, E.: Meteorological effects of the solar eclipse of 11 August 1999, Weather, 55, 430-446, doi:10.1002/j.14778696.2000.tb06481.x, 2000.

Hong, S.-Y., Dudhia, J., and Chen, S.-H.: A Revised Approach to Ice Microphysical Processes for the Bulk Parameterization of Clouds and Precipitation, Mon. Weather Rev., 132, 103-120, doi:10.1175/1520-0493(2004)132<0103:ARATIM>2.0.CO;2, 2004.

Hong, S.-Y., Noh, Y., and Dudhia, J.: A New Vertical Diffusion Package with an Explicit Treatment of Entrainment Processes, Mon. Weather Rev., 134, 2318-2341, doi:10.1175/MWR3199.1, 2006.

Iacono, M. J., Delamere, J. S., Mlawer, E. J., Shephard, M. W., Clough, S. A., and Collins, W. D.: Radiative forcing by long-lived greenhouse gases: Calculations with the AER radiative transfer models, J. Geophys. Res., 113, D13103, doi:10.1029/2008JD009944, 2008.

Kain, J. S.: The Kain-Fritsch Convective Parameterization: An Update, J. Appl. Meteorol., 43, 170-181, doi:10.1175/15200450(2004)043<0170:TKCPAU>2.0.CO;2, 2004.

Meeus, J. H.: Astronomical Algorithms, Willmann-Bell, Incorporated, Virginia, 1991.

Montornès, A., Codina, B., and Zack, J. W.: A discussion about the role of shortwave schemes on real WRF-ARW simulations. Two case studies: cloudless and cloudy sky, Tethys, 12, 13-31, doi:10.3369/tethys.2015.12.02, 2015.

Ohmura, A., Gilgen, H., Hegner, H., Müller, G., Wild, M., Dutton, E. G., Forgan, B., Fröhlich, C., Philipona, R., Heimo, A., König-Langlo, G., McArthur, B., Pinker, R., Whitlock, C. H., and Dehne, K.: Baseline Surface Radiation Network (BSRN/WCRP): New Precision Radiometry for Climate Research, B. Am. Meteorol. Soc., 79, 2115-2136, doi:10.1175/1520-0477(1998)079<2115:BSRNBW>2.0.CO;2, 1998.

Paltridge, G. W. and Platt, C. M.: Radiative processes in meteorology and climatology, Elsevier Scientific Publishing Company, Amsterdam-Oxford-New York, 1976.
Paulson, C. A.: The Mathematical Representation of Wind Speed and Temperature Profiles in the Unstable Atmospheric Surface Layer, J. Appl. Meteorol., 9, 857-861, doi:10.1175/15200450(1970)009<0857:TMROWS>2.0.CO;2, 1970.

Poli, P., Healy, S. B., and Dee, D. P.: Assimilation of Global Positioning System radio occultation data in the ECMWF ERAInterim reanalysis, Q. J. Roy. Meteor. Soc., 136, 1972-1990, doi:10.1002/qj.722, 2010.

Randhawa, J. S.: Mesospheric ozone measurements during a solar eclipse, J. Geophys. Res., 73, 493-495, doi:10.1029/JB073i002p00493, 1968.

Segal, M., Turner, R. W., Prusa, J., Bitzer, R. J., and Finley, S. V.: Solar Eclipse Effect on Shelter Air Temperature, B. Am. Meteorol. Soc., 77, 89-99, doi:10.1175/15200477(1996)077<0089:SEEOSA>2.0.CO;2, 1996.

Stewart, R. B. and Rouse, W. R.: Radiation and Energy Budgets at an Arctic Site during the Solar Eclipse of July 10, 1972, Artic Alpine Res., 6, 231, doi:10.2307/1550088, 1974.

Stull, R.: An Introduction to Boundary Layer Meteorology, Springer, the Netherlands, 1988.

Subrahamanyam, D. and Anurose, T.: Solar eclipse induced impacts on sea/land breeze circulation over Thumba: A case study, J. Atmos. Sol. Terr. Phy., 73, 703-708, doi:10.1016/j.jastp.2011.01.002, 2011.

Subrahamanyam, D., Anurose, T. J., Mohan, M., Santosh, M., Kiran Kumar, N. V. P., Sijikumar, S., Prijith, S. S., and Aloysius, M.: Atmospheric Surface-Layer Response to the Annular Solar Eclipse of 15 January 2010 over Thiruvananthapuram, India, Bound.-Lay. Meteorol., 141, 325-332, doi:10.1007/s10546-0119627-z, 2011.

Szałowski, K.: The effect of the solar eclipse on the air temperature near the ground, J. Atmos. Sol. Terr. Phy., 64, 1589-1600, doi:10.1016/S1364-6826(02)00134-7, 2002.

Tewari, M., Chen, F., Wang, W., Dudhia, J., LeMone, M. A., Mitchell, K., and Cuenca, R. H.: Implementation and verification of the unified NOAH land surface model in the WRF model, in: 20 th conference on weather analysis and forecasting/16th conference on numerical weather prediction, 11-15, 2004.

Vogel, B., Baldauf, M., and Fiedler, F.: The Influence of a solar eclipse on temperature and wind in the Upper-Rhine Valley - A numerical case study, Meteorol. Z., 10, 207-214, doi:10.1127/0941-2948/2001/0010-0207, 2001.

Webb, E. K.: Profile relationships: The log-linear range, and extension to strong stability, Q. J. Roy. Meteor. Soc., 96, 67-90, doi:10.1002/qj.49709640708, 1970.

Wu, J.-B., Wang, Z. F., Zhang, W., Dong, H. B., Pan, X. L., Li, J., Lin, C.-Y., and Xie, P. H.: The effects of a solar eclipse on photo-oxidants in different areas of China, Atmos. Chem. Phys., 11, 8075-8085, doi:10.5194/acp-11-8075-2011, 2011.

Zanis, P., Zerefos, C., Gilge, S., Melas, D., Balis, D., Ziomas, I., Gerasopoulos, E., Tzoumaka, P., Kaminski, U., and Fricke, W.: Comparison of measured and modeled surface ozone concentrations at two different sites in Europe during the solar eclipse on August 11, 1999, Atmos. Environ., 35, 4663-4673, doi:10.1016/S1352-2310(01)00116-9, 2001.

Zerefos, C., Balis, D., Zanis, P., Meleti, C., Bais, A., Tourpali, K., Melas, D., Ziomas, I., Galani, E., Kourtidis, K., Papayannis, A., and Gogosheva, Z.: Changes in surface UV solar irradiance and ozone over the balkans during the eclipse of August 
11, 1999, Adv. Space Res., 27, 1955-1963, doi:10.1016/S02731177(01)00279-4, 2001.

Zerefos, C. S., Gerasopoulos, E., Tsagouri, I., Psiloglou, B. E., Belehaki, A., Herekakis, T., Bais, A., Kazadzis, S., Eleftheratos, C., Kalivitis, N., and Mihalopoulos, N.: Evidence of gravity waves into the atmosphere during the March 2006 total solar eclipse, Atmos. Chem. Phys., 7, 4943-4951, doi:10.5194/acp-74943-2007, 2007.
Zhang, D. and Anthes, R. A.: A High-Resolution Model of the Planetary Boundary Layer - Sensitivity Tests and Comparisons with SESAME-79 Data, J. Appl. Meteorol., 21, 1594-1609, doi:10.1175/1520-0450(1982)021<1594:AHRMOT>2.0.CO;2, 1982. 